Supporting Information

\title{
One-Step, Continuous Three-Dimensional Printing of Multi-Stimuli-Responsive Bilayer Microactuators via a Double-Barreled Theta Pipette
}

Xiao Huan, Sanghyeon Lee, Heekwon Lee, Zhaoyi Xu, Jihyuk Yang, Mojun Chen, Yu Liu, and

Ji Tae Kim*

Department of Mechanical Engineering, The University of Hong Kong, Pokfulam Road, Hong

Kong, China

*Corresponding author. Email: jtkim@,hku.hk 


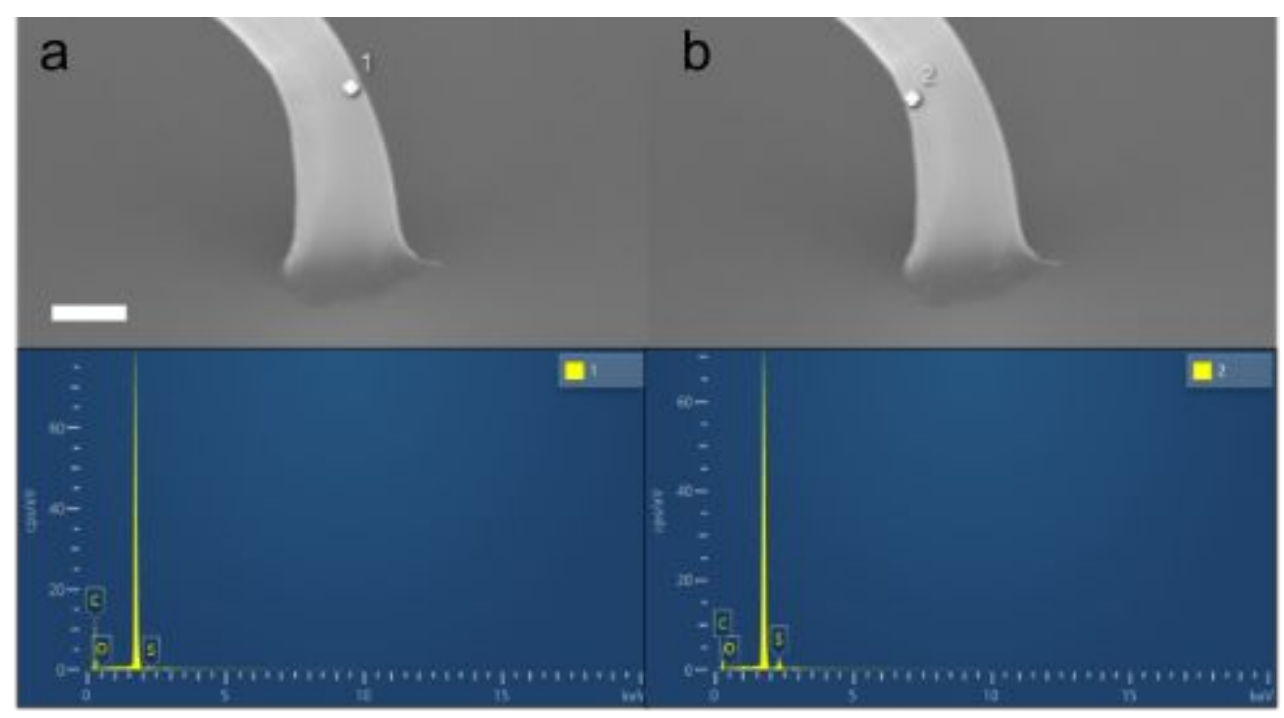

Figure S1. Quantitative analysis of energy-dispersive X-ray spectroscopy images (Scale bar: $2 \mu \mathrm{m})$.

(a) Composition of point 1 on the polystyrene layer. (b) Composition of point 2 on the poly(3,4ethylenedioxythiophene): polystyrene sulfonate layer.

\begin{tabular}{|l|l|l|}
\hline Sulfur/Carbon ratio & Measured ratio & Calculated ratio \\
\hline PEDOT:PSS & 0.341 & 0.307 \\
\hline PS & 0 & 0.003 \\
\hline
\end{tabular}

Table S1. Content ratio of sulfur and carbon in poly(3,4-ethylenedioxythiophene):polystyrene sulfonate (PEDOT:PSS) and polystyrene (PS) layers. 

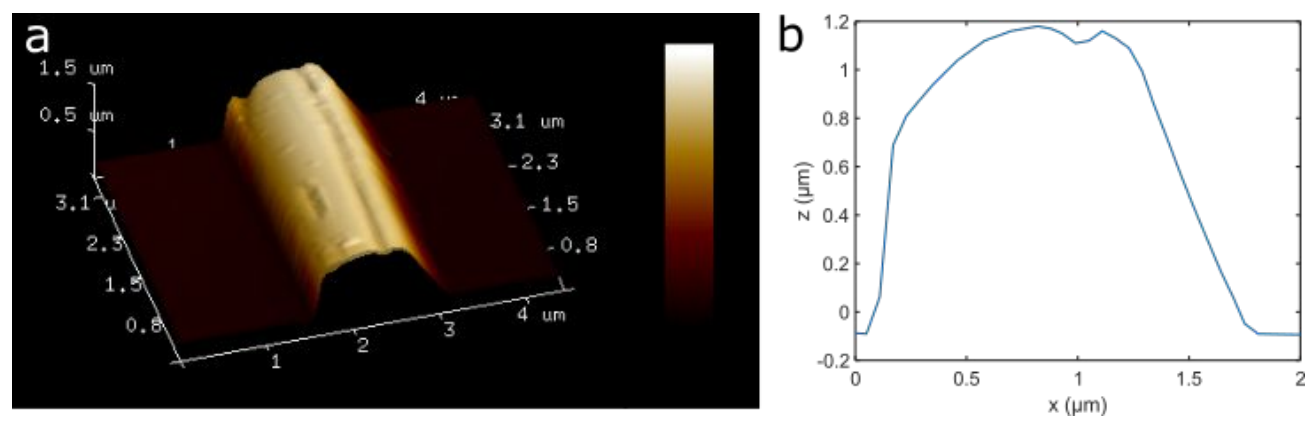

Figure S2. Atomic force microscopy images of (a) the surface of the bilayer structure, and (b) a cross section of this structure.

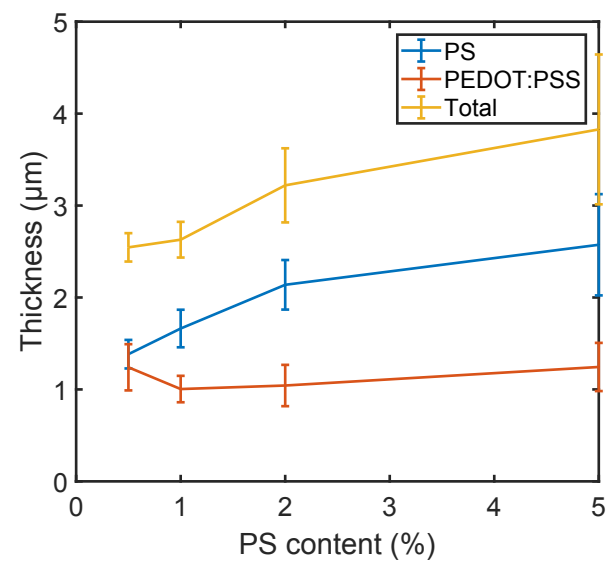

Figure S3. Effects of polystyrene (PS) ink content on the layer thickness. The thickness of the PS layer increases from 1.38 to $2.57 \mu \mathrm{m}$ as the PS ink content increases from $0.5 \%$ to $5 \%$, while the thickness of the poly(3,4-ethylenedioxythiophene):polystyrene sulfonate (PEDOT:PSS) layer remains almost constant. 


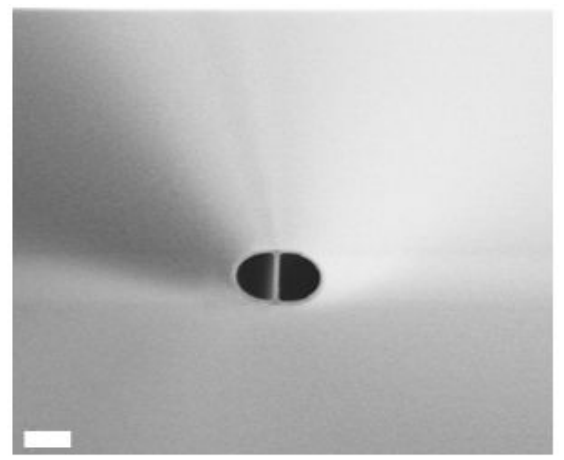

Figure S4. Field-emission scanning electron microscopy image of a glass double-barreled theta pipette with a barrel-aperture diameter of $\sim 2 \mu \mathrm{m}$ (scale bar: $1 \mu \mathrm{m}$ ).

a

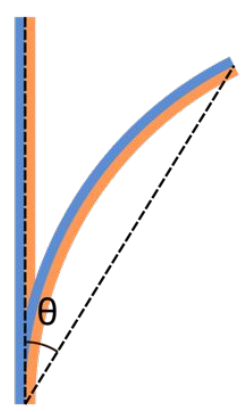

b

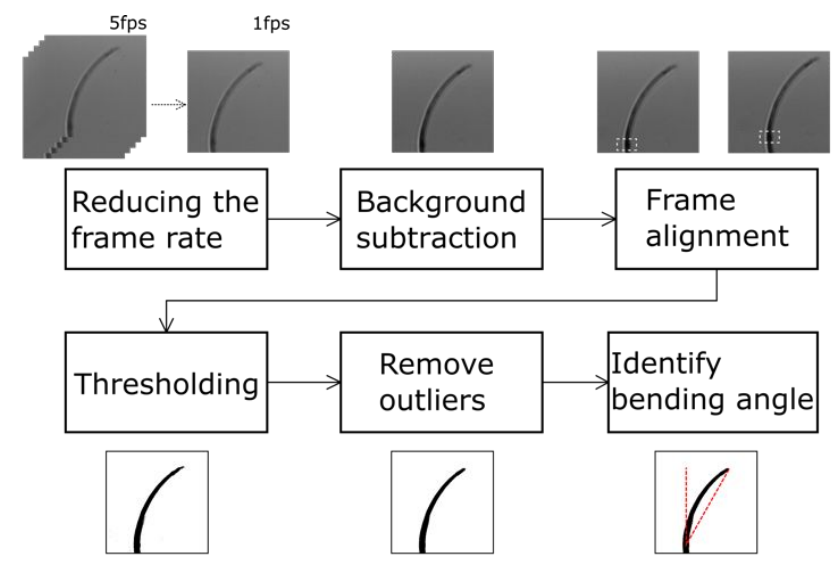

Figure S5. (a) Definition of bending angle. (b) Flow chart of image processing and bending angle identification. The images were obtained by a high-magnification objective (Mitutoyo, $50 \times, \mathrm{f}=$ 200 ) and detected by a CMOS camera (Thorlabs, frame rate 5 fps, $1280 \times 1024$ pixels). The pixel resolution was $0.12 \mu \mathrm{m} / \mathrm{pixel}$. The frame rate of the recorded images was reduced to $1 \mathrm{fps}$ to be processed faster. After background subtraction, alignment, noise removing and thresholding, the 
images were transferred into binary images, from which a series of bending angles $(\theta)$ could be calculated.
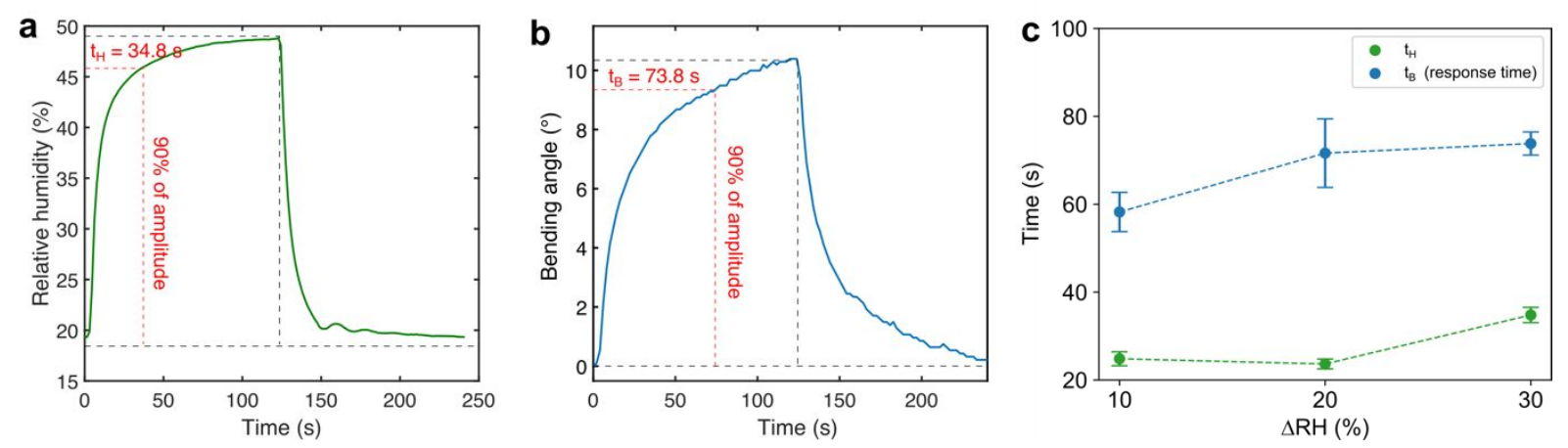

Figure S6. Single bending cycle analysis. (a) Single pulse of RH switching with an amplitude of $30 \%(\Delta \mathrm{RH}=30 \%)$ and a frequency of $4.17 \mathrm{mHz}\left(\mathrm{t}_{\mathrm{H}}\right.$ : time for reaching $90 \%$ of $\left.\Delta \mathrm{RH}\right)$. (b) Single bending cycle of a freestanding PS/PEDOT:PSS bilayer microwire upon corresponding RHswitching $\left(\mathrm{t}_{\mathrm{B}}\right.$ : response time, defined as the time for reaching $90 \%$ of its maximum bending displacement). (c) Dependences of $t_{B}$ (blue) and $t_{H}$ (green) on $\Delta R H$.

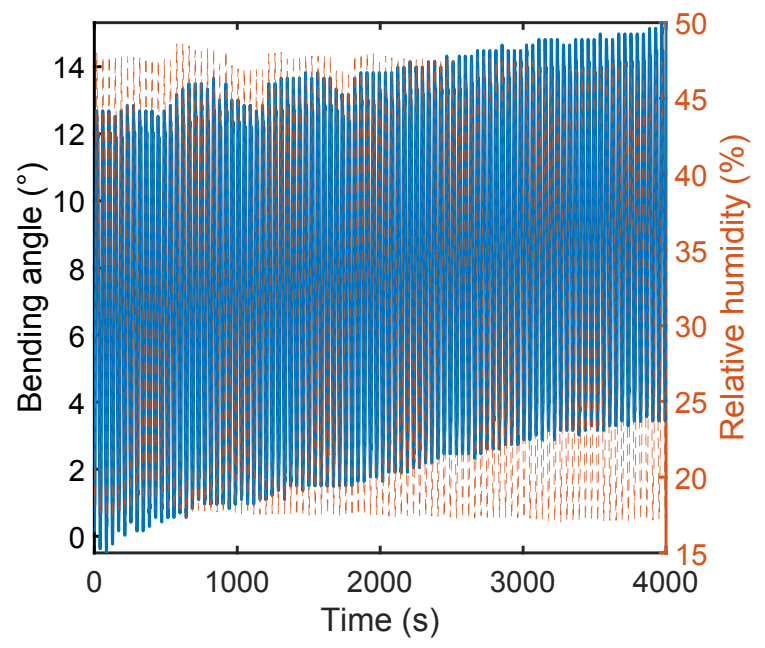


Figure S7. Cyclic stability of the bending angle of a bilayer structure over 4,000 s (20\%-50\%).

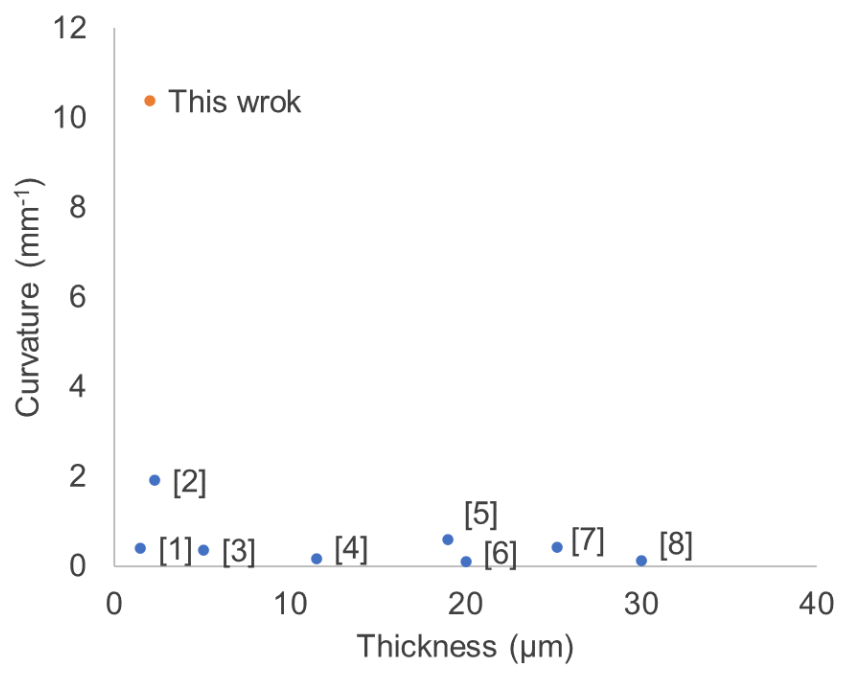

Figure S8. Comparison of bending curvature of our actuators to those of other humidity-responsive actuators. ${ }^{1-8}$ The materials and dimensions of these actuators are shown in Table S2. 


\begin{tabular}{|c|c|c|}
\hline Reference & Materials & Dimensions/ $\mu \mathrm{m}$ \\
\hline This work & $\begin{array}{l}\text { Polystyrene/poly( } 3,4- \\
\text { ethylenedioxythiophene):polystyrene } \\
\text { sulfonate }\end{array}$ & $2 \times 50 \times 2$ \\
\hline [1] & Bacillus subtilis spores/silicon & $30 \times 300 \times 1.5$ \\
\hline [2] & $\begin{array}{l}\text { Graphene oxide }(\mathrm{GO}) / \text { reduced graphene } \\
\text { oxide (rGO) }\end{array}$ & $1,000 \times 3,000 \times 2.3$ \\
\hline [3] & rGO/polypyrrole (PPy) & $1,500 \times 16,000 \times 5.1$ \\
\hline [4] & $\mathrm{GO} / \mathrm{rGO}$ & $1,000 \times 12,000 \times 11.5$ \\
\hline [5] & Liquid-crystalline polymer/polyamide- 6 & Thickness: 19 \\
\hline [6] & GO/poly(methyl methacrylate) & $1,000 \times 10,000 \times 20$ \\
\hline [7] & GO/PPy & $2,000 \times 15,000 \times 25.2$ \\
\hline [8] & $\mathrm{GO} / \mathrm{rGO}$ & $30 \times 20,000 \times 30$ \\
\hline
\end{tabular}

Table S2. Materials and dimensions of humidity-responsive actuators. 


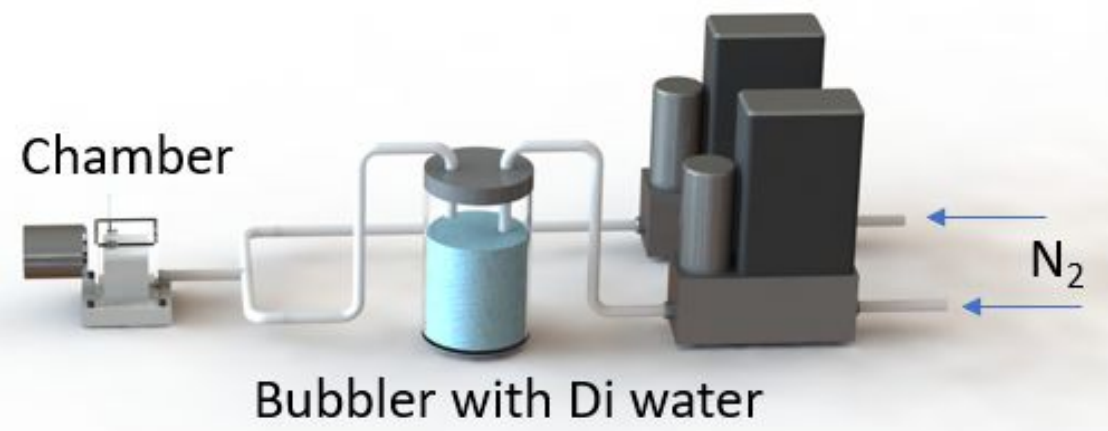

Figure S9. The setup for humidity control ( $\mathrm{Di}=$ deionized).

\section{REFERENCES}

1. Chen, X.; Mahadevan, L.; Driks, A.; Sahin, O., Bacillus Spores as Building Blocks for Stimuli-Responsive Materials and Nanogenerators. Nat. Nanotechnol. 2014, 9 (2), 137-141.

2. Xu, G.; Chen, J.; Zhang, M.; Shi, G., An Ultrasensitive Moisture Driven Actuator Based on Small Flakes of Graphene Oxide. Sensors Actuators B: Chem. 2017, 242, 418-422.

3. Jiang, Y.; Hu, C.; Cheng, H.; Li, C.; Xu, T.; Zhao, Y.; Shao, H.; Qu, L., Spontaneous, Straightforward Fabrication of Partially Reduced Graphene Oxide-Polypyrrole Composite Films for Versatile Actuators. ACS Nano 2016, 10 (4), 4735-4741. 
4. Han, D.-D.; Zhang, Y.-L.; Liu, Y.; Liu, Y.-Q.; Jiang, H.-B.; Han, B.; Fu, X.-Y.; Ding, H.; Xu, H.-L.; Sun, H.-B., Bioinspired Graphene Actuators Prepared by Unilateral UV Irradiation of Graphene Oxide Papers. Adv. Funct. Mater. 2015, 25(28), 4548-4557.

5. Dai, M.; Picot, O. T.; Verjans, J. M. N.; de Haan, L. T.; Schenning, A. P. H. J.; Peijs, T.; Bastiaansen, C. W. M., Humidity-Responsive Bilayer Actuators Based on a Liquid-Crystalline Polymer Network. ACS Appl. Mater. Interfaces 2013, 5(11), 4945-4950.

6. Gao, Y.-Y.; Zhang, Y.-L.; Han, B.; Zhu, L.; Dong, B.; Sun, H.-B., Gradient Assembly of Polymer Nanospheres and Graphene Oxide Sheets for Dual-Responsive Soft Actuators. ACS Appl. Mater. Interfaces 2019, 11 (40), 37130-37138.

7. Dong, Y.; Wang, J.; Guo, X.; Yang, S.; Ozen, M. O.; Chen, P.; Liu, X.; Du, W.; Xiao, F.; Demirci, U.; Liu, B.-F., Multi-Stimuli-Responsive Programmable Biomimetic Actuator. Nat. Commun. 2019, $10(1), 4087$.

8. Cheng, H.; Liu, J.; Zhao, Y.; Hu, C.; Zhang, Z.; Chen, N.; Jiang, L.; Qu, L., Graphene Fibers with Predetermined Deformation as Moisture-Triggered Actuators and Robots. Angew. Chem. Int. Ed. 2013, 52 (40), 10482-10486. 
S-1 